\title{
UNSUPERVISED CALIBRATED SONAR IMAGING FOR SEABED OBSERVATION USING HIDDEN MARKOV RANDOM FIELDS
}

\author{
R. Fablet and J.-M. Augustin \\ Ifremer, TSI-STH \\ Technople Brest Iroise, 29280 PLOUZANE, France
}

\begin{abstract}
This paper aims at enhancing seabed imaging issued from sonar systems. Such imaging systems produce maps of backscattering strength relative to physical seabed characteristics. However, these maps show a dependency on the incident angles. This dependency is in addition seabed-related. To enhance the quality of these imaging systems, we develop an unsupervised approach to compensate for these seabed-related angular dependencies. Our approach combines robust estimation and hidden Markov random fields. Results on real BS images demonstrate the relevance of our approach to improve seabed observation.
\end{abstract}

\section{PROBLEM STATEMENT AND RELATED WORK}

Sonar imaging provides a remote sensing tool to observe and characterize the physical properties of the seafloor and is increasingly exploited for a variety of applications such as environmental monitoring, marine geosciences and biology, as well as oil industry or defense. The current challenge for swath sonars from imaging systems lies in the calibration of reliable instruments for reflectivity measurement. This is requirement both to make feasible the comparison of seafloor properties at different locations along a single observation path as well as the monitoring of the changes of seafloor properties along time.

This issue comes from the intrinsic characteristics of sonar imaging systems. Since these systems rely on acoustic reflection of echoes emitted by the system, reflectivity measurement of the seafloor obviously depends on the incident angle. Consequently, similar seafloor types viewed from different incident angles do not produce similar reflectivity measurement. Sonar calibration is then a major task to compensate for this angular dependencies. This issue is indeed enhanced by the fact that these dependencies are seafloor-related. Hence, the calibration issue is hardly linked to seafloor-based segmentation.

This paper tackles this calibration issue and exploit robust estimation of backscattering models and hidden Markov models to state this issue within a Bayesian framework. The paper is organized as follows. Section 2 deals with Backscat- ter modelling and estimation, and Section 3 presents the proposed unsupervised approach for sonar calibration. Experimental results are reported in Section 4.

\section{BACKSCATTER MODELLING AND ESTIMATION}

\subsection{Characteristics of acoustic signals recorded by sonar systems}

Signals recorded by seafloor-mapping swath sonars potentially provide an absolute measurement of the seafloor backscattering strength as a function of incident angle. However the measured echo level is not only dependent on the seafloor backscattering strength, but is actually affected by phenomena bound to the measurement configuration (transmission range and angle), to seawater properties (absorption, refraction) and to the sonar itself (array directivity patterns, receiver processing). These various factors may be summarized in the classical sonar equation [5], expressed here in $\mathrm{dB}$, summarizing the building of the echo level as a function of time:

$$
\begin{aligned}
E L(t) & =S L+D t\left[\theta_{a}(t)\right]-2 T L[R(t)]+B S\left[\theta_{b}(t)\right] \\
& +10 \log \left(S\left[R(t), \theta_{b}(t)\right]\right)+\operatorname{Dr}\left[\theta_{a}(t)\right]+P G(t)
\end{aligned}
$$

$E L(t)$ is the electrical signal (in $\mathrm{dB}$ re. $1 \mathrm{~V}$ ) corresponding to the echo level, as a function of reception time $t . S L$ is the source level (dB re.1Pa@1m), modulated by the transmission directivity pattern $D t$ expressed as a function of angle at the sonar arrays $\theta_{a}(t) . R(t)$ is the range/time relation, and $T L$ the Transmission loss. The backscattering strength $B S$, seafloor dependent, also strongly depends on the signal frequency and moreover on the incident angle $\theta_{b}(t)$ on the seafloor. Scattering area $S$ is bound both to the sonar parameters (beam aperture, signal duration) and the range/angle/time relations. $D r$ is the array directivity patterns on reception. Finally, $P G$ is the sonar receiver processing gain featuring both constant terms (linked to various stages of pre-amplification and filtering) and a time variation.

Among these different components of the received echo level, all except the backscattering strength $B S$ are mainly related to the characteristics of the exploited sonar system. Previous work $[1,4]$ have proposed post-processing steps to deal 

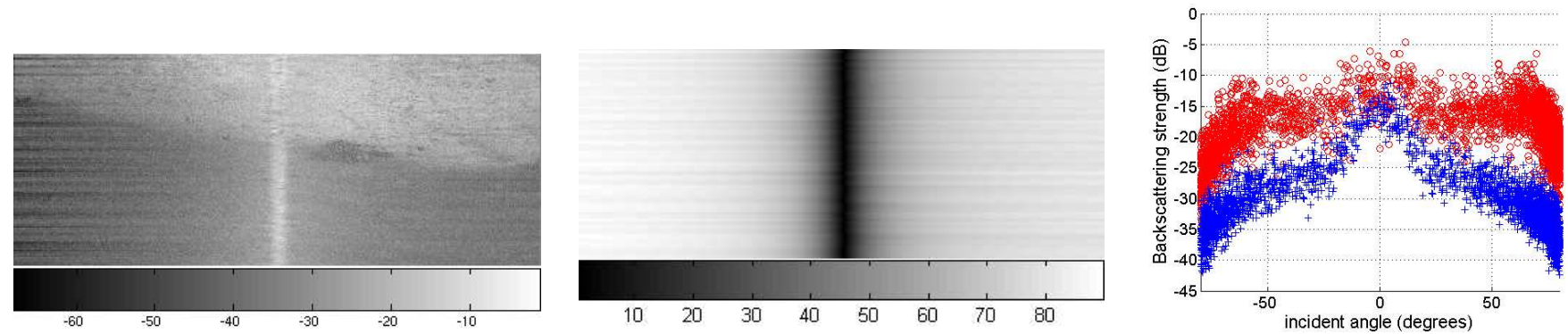

Fig. 1. Illustration of the characteristics of BS images for seabed imaging: from left to right, BS image involving two seafloor types; associated map of incident angles; plot of the BS measure as a function of the incident angles for both seaflor types.

with these components. Fig.1 displays an example of such a post-processed BS image. This image conveys meaningful information about the physical properties of the seafloor. However, as noted previously and stressed by Fig.1, this measure is dependent on the incident angle. More precisely, BS values are characterized by a specular component (high values) for incident angles close to 0 degree and decrease when the incident angle increases. Consequently, the same seafloor region viewed at different incident angles does not resort to the same BS map. The need to compensating for this bias is obvious. It is however a difficult task since the relation between the BS value and the incident angle is seafloor dependent as illustrated by Fig.1.

\subsection{BS modeling}

A variety of models have been proposed [5] to model for a given seafloor the evolution of the mean BS measure as a function of the incident angle. In this work, we rely on a trade-off between the complexity of the model and its ability to account for a wide range of seafloor configurations. Following [5], the considered model is stated as the sum of a specular component and of a generalized Lambert's law:

$B S_{\Psi}(\theta)=10 \log _{10}\left(10^{-A} \exp \left(-B \theta^{2}\right)+10^{-C} \cos ^{D}(\theta)\right)$

In the subsequent, $\Psi$ will denote the set of model parameters $(A, B, C, D)$.

\subsection{Robust sequential estimation}

Let us assume that we are given with a set of BS values $\left\{B S_{i}\right\}_{i}$ associated with incident angles $\left\{\theta_{i}\right\}_{i}$. We further assume that $\tau_{S i}$ is the likelihood that the BS measure $B S_{i}$ is relative to a given seafloor type $S$. Hence, the estimation of the BS model $\Psi_{S}$ for seafloor type $S$ is stated as the minimization of the following robust criterion:

$$
\widehat{\Psi}_{S}=\arg \min _{\Psi_{S}} \sum_{i} \tau_{S i} \cdot \rho\left[B S_{i}-B S_{\Psi_{S}}\left(\theta_{i}\right)\right]
$$

rho is a robust function, such as the Leclerc estimator [3]: $\rho(r)=1-\exp \left(-r^{2} / \sigma_{R}^{2}\right)$, where $\sigma$ is a scale parameter.
Equivalently, this robust minimization is solved for using an iterated least-square procedure. This procedure iterates two steps: 1) the computations of robust weights for the current estimate $\Psi_{S}$ :

$$
\omega_{i}=\phi\left(B S_{i}-B S_{\Psi_{S}}\left(\theta_{i}\right)\right)
$$

where $\phi$ is the influence function $\phi(r)=\rho^{\prime}(r) / r$;2) the minimization of the following quadratic criterion for the updated robust weights $\omega_{i i}$ :

$$
\widehat{\Psi}_{S}=\arg \min _{\Psi_{S}} \sum_{i} \tau_{S i} \cdot \omega_{i} \cdot\left[B S_{i}-B S_{\Psi_{S}}\left(\theta_{i}\right)\right]^{2}
$$

Rather than directly solving for this quadratic criterion using a gradient-based or incremental approach, we adopt a sequential scheme. It comes to alternately updating specular and Lambert components. More precisely, for given parameters of the specular component, the parameters of the Lambert component are updated as the solution of the following criterion:

$$
\widehat{\Psi}_{L, S}=\arg \min _{\Psi_{L, S}} \sum_{i} \tau_{S i} \cdot \omega_{i} \cdot\left[\widetilde{B S}_{i}^{s p e c}-B S_{\Psi_{L, S}}\left(\theta_{i}\right)\right]^{2}
$$

where $\Psi_{L, S}$ is the BS model issued from the Lambert component of model $\Psi_{S}, \widetilde{B S}_{i}^{s p e c}$ the residual when compensating for the current estimate of the specular component. Since model $\Psi_{L, S}$ is linear w.r.t. parameters $C$ and $D$, the updated model parameters $\widehat{\Psi}_{L, S}$ are exactly computed as a leastsquare solution:

$$
\widehat{\Psi}_{L, S}=\left[\sum_{i} \tau_{S i} \omega_{i} Z_{i}^{T} Z_{i}\right]^{-1} \sum_{i} \tau_{S i} \omega_{i} \widetilde{B S}_{i}^{\text {spec }} Z_{i}
$$

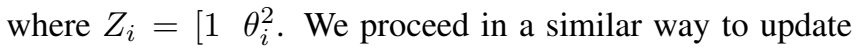
the parameters of the specular component given the Lambert component. We iterate this robust sequential estimation until convergence.

To improve the convergence properties of this robust estimation scheme, scale parameter $\sigma_{R}$ is set to $1.57 * \operatorname{med}\left(\left\{r_{i}\right\}\right)$, where $\left\{r_{i}\right\}$ are the residuals relative to the initial least-square parameter estimate. This scale parameter is hen decreased according to a geometrical law. 


\section{BAYESIAN COMPENSATION FOR SEABED-RELATED ANGULAR DEPENDENCIES}

We aim at removing the effects of the incident angles in the measure of the backscattering strength. Since these effects are seabed-related, this compensation can be regarded as a segmentation issue w.r.t. seabed types. More precisely, given a set of seabed types $\left\{S_{1}, \ldots, S_{n}\right\}$, our goal is to estimate the posterior likelihood of the map of the seabed types given the observed BS image. Let us denote by $x$ the map of the seabed types and $B S$ the map of BS measures associated with the map of incident angles $\theta$, we aim at evaluating the following posterior likelihood for any pixel $p$ and seabed type $S$ :

$$
\tau_{p, S}=P\left(x_{p}=S \mid B S ; \theta ; x_{q}, q \neq p\right)
$$

Given these posterior likelihoods, the compensated BS image can be estimated as:

$$
B S_{c o m p}(p)=B S_{p}-\sum_{k} \tau_{p, S_{k}}\left(B S_{\Psi_{S_{k}}}\left(\theta_{p}\right)-C_{S_{k}}\right)
$$

where $\sum_{k} \tau_{p, S_{k}}\left(B S_{\Psi_{S_{k}}}\left(\theta_{p}\right)-C_{S_{k}}\right)$ stands for the expectation of the angular dependency, when the reference BS level is set to the value of Lambert's component at incident angle 0 .

\subsection{Markovian setting and Gibbs sampling}

In order to evaluate posterior likelihoods $\tau_{p, S}$, we resort to a Markovian setting [2]. Assuming that $x$ is a Markov random field with a 4-neighborhood structure and that BS measures $B S_{p}$ are independent conditionally to $x_{p}, \tau_{p, S}$ can be written as:

$$
\tau_{p, S} \propto P\left(B S_{p} \mid x_{p}=S, \theta_{p}\right) P\left(x_{p}=S \mid x_{q}, q \neq p\right)
$$

Data-driven $P\left(B S_{p} \mid x_{p}=S, \theta_{p}\right)$ term is evaluated as the Gaussian likelihood of residual $B S_{p}-B S_{\Psi_{S_{k}}}\left(\theta_{p}\right)$ :

$$
P\left(B S_{p} \mid x_{p}=S, \theta_{p}\right)=\frac{\exp \left(-\frac{\left(B S_{p}-B S_{\Psi_{S}}\right)^{2}}{2 \sigma_{S}^{2}\left(\theta_{p}\right)}\right)}{\sqrt{2 \pi \sigma_{S}^{2}\left(\theta_{p}\right)}}
$$

where $\sigma_{S}\left(\theta_{p}\right)$ is the angle-dependent standard deviation of the Gaussian error model for seabed type $S$. Let us stress that the data-driven model involves an angle-dependent standard deviation, since it has been observed experimentally that the distribution of the residual errors vary over angles.

The a priori Markovian model set on $x$ comes to:

$$
\begin{aligned}
P\left(x_{p} \mid x_{q}, q \neq p\right) & =P\left(x_{p} \mid x_{q}, q \in V_{p}\right) \\
& \propto \exp \left[-\gamma \sum_{q \in V_{p}} \delta\left(x_{p}-x_{q}\right)\right]
\end{aligned}
$$

where $V_{p}$ are the four neighbors of pixel $p, \delta$ the Dirac function and $\gamma$ the regularization coefficient which balances the relative influences of the regularization term and of the datadriven term.

The overall model is then parametrized by the set of seabed BS models $\left\{\Psi_{S}, \sigma_{S}\right\}$ and the regularization weight $\gamma$. Given these parameters, posterior likelihoods $\tau_{p, S}$ are estimated using the Gibbs sampler [2]. From an initial label map $x^{0}$, it comes to generate a sequence of maps $\left\{x^{k}\right\}$. At iteration $k$, $x^{k}$ is first initialized to $x^{k-1}$. Then, for each pixel $p$, seabed label $x^{k}(p)$ is randomly selected within $\left\{S_{1}, \ldots, S_{N}\right\}$ according to the likelihoods $P\left(x_{p}^{k}=S \mid B S ; \theta ;\left\{x_{q}^{k}, q \in V_{p}\right\}\right)$ given by Eq. 10. From the sequence of maps $\left\{x^{k}\right\}$, posterior $\tau_{p, S}$ is estimated as an empirical average:

$$
\tau_{p, S}=\frac{\#\left\{k \in[N 1, N 2], x^{k}(p)=S\right\}}{N 2-N 1+1}
$$

where \#A is the cardinality of set $A . N 2$ is the length of the sequence generated by the Gibbs sampler and $N_{1}$ the number of iterations needed to reach the stationarity.

\subsection{Unsupervised compensation}

The compensation scheme defined by Eq. 9 as well as the Gibbs sampler require to explicitly define the BS models. Assuming that the number of seabed types is known, the compensation is then an unsupervised issue where we need to jointly achieve the estimation of the parameters of the BS models and the compensation. To solve for this unsupervised issue, we exploit an ICE (Iterative Conditional Estimation) procedure [6]. At iteration $k$, this procedure involves two steps:

- step 1 : For current model parameters $\left\{\Psi_{S}^{k}, \sigma_{S}^{k}\right\}$, posteriors $\tau_{p, S}^{k}$ are estimated using the Gibbs sampler (cf. Eq.13). Note that, as initialization, we provide the last map of the Gibbs sequence generated at iteration $k-1$.

- step 2 : Model parameters $\left\{\Psi_{S}^{k}, \sigma_{S}^{k}\right\}$ are updated from current posterior estimates $\tau_{p, S}^{k}$ as stated by Eq.7. Following a non-parametric approach, standard deviation models $\sigma_{S}^{k}$ are updated as weighted averages of the current residuals, with weights computed as the products of the posteriors and a Gaussian angular kernel to account for angular dependencies.

In order to achieve a better convergence of the Gibbs sampler, we adopt a multiresolution strategy. From a Gaussian pyramid of the Initial BS and angular maps, we iterate the ICE procedure from the coarsest resolution to the finest one. The initialization of the Gibbs sampler at each resolution is issued from the last seabed map generated at the previous resolution.

At the coarsest resolution, the initial model parameters $\left\{\Psi_{S}^{0}, \sigma_{S}^{0}\right\}$ are given by the EM-based estimation of the parameter of a mixture models of BS models. In addition, to 

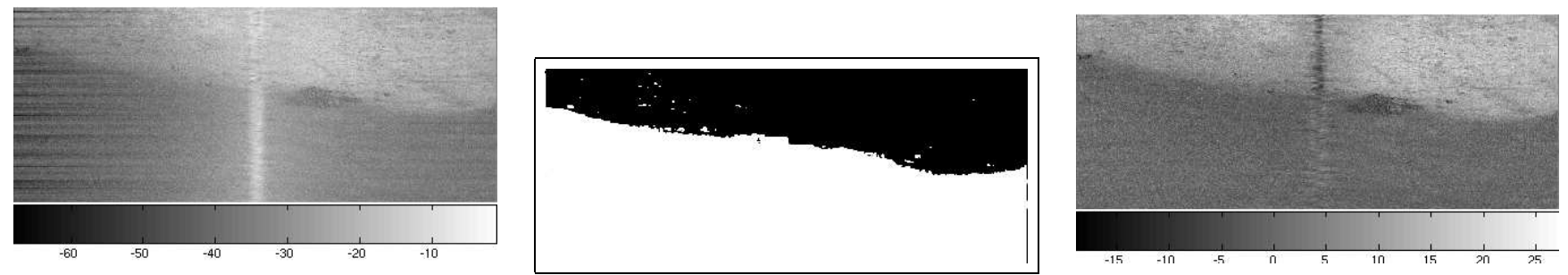

Fig. 2. Example of unsupervised BS (Backsctarreing Strength) compensation: from left to right, original BS map involving two seafloor types, segmentation map issued from the estimated posterior likelihood, compensated BS image.

increase the robustness of the proposed scheme, the first ICE steps are carried out with simpler BS models accounting only Lambert's components. The specular components are introduced after a predefined number of iterations.

At final iteration and finest resolution, the compensated BS map is finally computed from Eq. 9 using the final posteriors $\tau_{p, S}^{K}$.

\section{EXPERIMENTS}

We have carried out experiments with real BS observations acquired with a multibeam sonar system. We report in Fig. 2 an example including the original images, the segmentation map given by $\widehat{x}_{p}=\arg \max _{S} \tau_{p, S}$ and the compensated BS map. This two example shows how the proposed unsupervised compensation scheme can improve the visualization of seafloor characteristics. In both cases, the specular component is clearly identified by the very bright vertical zone corresponding to low incident angles, whereas regions on both sides of the image (i.e., relative to greater incident angles) tend to be darker. The reported segmentation map illustrates that the proposed unsupervised scheme succeeds in identifying the meaningful seafloor models. The estimated BS models are displayed in Fig.3. The compensated BS image permits to better visualize and compare the reflectivity measures within and between each seafloor region.

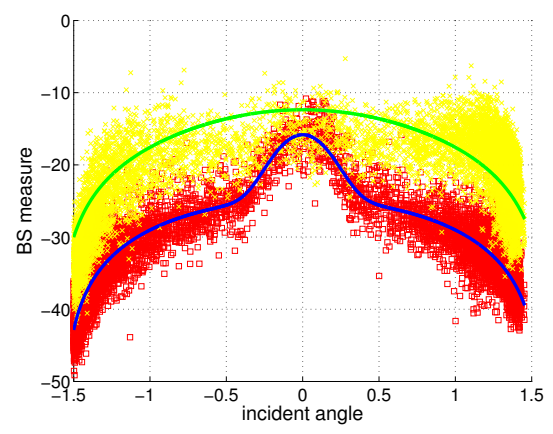

Fig. 3. Estimated BS models for the sonar image displayed in Fig.2: each color refers to one model with the associated BS data.
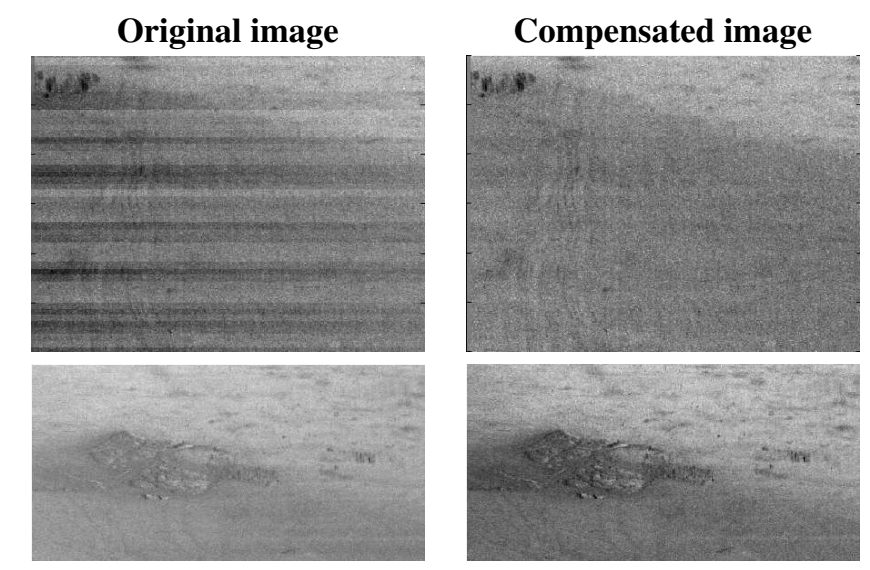

Fig. 4. Comparison of the original BS map and of the compensated one for two subregions of the example reported in Fig. 2.

The zoom on two regions of the BS map, displayed in Fig;4 further stresses the enhancement brought by our scheme. As an additional relevant feature, it also removes artifacts, appearing as horizontal lines, which are due variations of the incident angles along the path of the sonar systems caused by pitch and roll. Since the proposed compensation produces calibrated reflectivity measures, these observation artifacts are removed.

\section{REFERENCES}

[1] J. M. Augustin and X. Lurton. Image amplitude calibration and processing for seafloor mapping sonars. In Proc. of IEEE on Ocean, Oceans'05, Brest, France, June 2005.

[2] S. Geman and D. Geman. Stochastic relaxation, Gibbs distribution and the Bayesian restoration of images. IEEE Trans. on Pattern Analysis and Machine Intelligence, 6(6):721-741, 1984.

[3] P. Huber. Robust Statistics. John Wiley \& Sons, 1981.

[4] X.Lurton L.Hellequin, J.M.Boucher. Processing of high-frequency multibeam echo sounder data for seafloor characterization. IEEE Journal of Oceanic Engineering, 28(1):78-89, 2003.

[5] X. Lurton. An introduction to underwater acoustics. Springer, 2002.

[6] F. Salzenstein and W. Pieczynski. Parameter estimation in hidden fuzzy markov random fields and image segmentation. Graph. Models: Image Process., 59(4):205-220, 1997. 